\title{
A Rare Case of Olecranon Bursitis caused by Mycobacterium abscessus Treated with Surgical Intervention
}

\author{
Sneha Bowalekar* and Rahul Gadkari
}

${ }^{1}$ Consultant Microbiologist, Dr. Jariwala Laboratory, 1 st floor, Rasraj Heights, Rokadia Lane, Near Gokul Hotel, Boriwali West, Mumbai, Maharashtra, India

${ }^{2}$ Consultant Orthopedic Surgeon, Sailee Hospital and Diagnostic Centre, Prathamesh Horizon, New Link Road, Boriwali West, Mumbai, Maharashtra, India

*Corresponding author

\section{A B S T R A C T}

Keywords

M. abscessus,

Olecranon bursitis, Immunocompetant

Article Info

Accepted:

07 January 2021

Available Online:

10 February 2021
The present case of Mycobacterium abscessus olecranon bursitis in an immunocompetant male treated with surgical drainage without requirement of antimycobacterial therapy. Nontuberculous mycobacteria are widely listed as an infective cause of olecranon bursitis and other skin and soft tissue infections. Direct inoculation of the organism during trauma leads to musculoskeletal infections in particular. We could not find any other case report of olecranon bursitis caused by $M$. abscessus in the literature. This case extends the view about nontuberculous mycobacterial infections in immunocometant adults with co-morbid condition such as diabetes.

\section{Introduction}

Nontuberculous mycobacteria (NTM) are increasingly being reported as cause of infections in immunocompromised as well as immunocompetant patients. They are ubiquitously present in the environment thus contributing to accidental inoculation and infection at pulmonary as well as extrapulmonary sites. Diagnosis of NTM infections takes a long course and advanced identification techniques have to be implemented for early reporting, clinical correlation and follow up. We report a case of olecranon bursitis caused by NTM identified as Mycobacterium abscessus. The infection was treated with surgical drainage of olecranon bursa fluid. Clinical follow up of patient was uneventful.

\section{Case report}

57 year old male, presented to an orthopedician with acute onset swelling and pain over left elbow. He was afebrile at presentation. Draining lymph node enlargement was not seen. He had history of fall one and half months back when he had 
injured his left elbow joint. He had taken symptomatic treatment from a general physician and improved. Later when the swelling developed, he again consulted the general practitioner who prescribed him with Tab. Augmentin 650mg BD for 10 days. The swelling continued to be the same despite the antibiotic treatment. The patient was then referred to the orthopedician who surgically drained the olecranon bursa fluid in minor OT. He was not prescribed with any antibiotics by the orthopedician. The fluid aspirated from olecranon bursa was sent to our laboratory for AFB Culture.

Specimen was $1.5 \mathrm{ml}$, reddish, thin purulent liquid. Primary smear findings were not significant. Gram stained smear showed presence of few pus cells with no organism. $\mathrm{ZN}$ staining was unremarkable for presence of acid and alchohol fast bacilli.

The fluid was treated with NALC-NAOH (4\%) for decontamination and then mixed with $50 \mathrm{ml}$ of sterile phosphate buffer solution (PBS) (pH 6.8) and centrifuged at $5500 \mathrm{rpm}$ for 15 minutes. Supernatant was decanted and the deposit was resuspended in PBS to achieve volume of $3 \mathrm{ml}$.

$500 \mu \mathrm{l}$ of decontaminated and concentrated specimen was inoculated in MGIT (Mycobacteria Growth Indicator Tube, Becton Dickinson) broth and 2-3 drops of treated specimen are inoculated on Lowenstein Jensen Medium. MGIT broth (Middelbrook 7H9 broth) 17 days after inoculation and incubation at $37^{\circ} \mathrm{C}$ showed a spectrophotometric reading of 20 (on BACTEC MicroMGIT instrument). The MGIT broth had turned turbid. LJ agar showed no growth after 42 days of incubation.

$\mathrm{ZN}$ staining from MGIT broth showed presence of Acid Fast Bacilli in clusters, palisades, cording noted at some places. The bacilli were slender, 6-8 $\mu \mathrm{m}$ in size, some filamentous. Simultaneously, subculture from MGIT broth was done on Blood Agar Plate and LJ agar.

Blood Agar plate incubated at $37^{\circ} \mathrm{C}$ showed shiny, grayish white growth after 4 days of incubation. Individual colonies after further incubation were small, round, about $3-4 \mathrm{~mm}$ in diameter, white non-pigmented, smooth, convex with defined margins. Gram Staining of growth revealed Gram positive bacteria approximately $6-8 \mu \mathrm{m}$ in size, arranged in needles. $\mathrm{ZN}$ staining carried out on same growth revealed Acid Fast bacilli 6-8 $\mu \mathrm{m}$ in size, arranged in cords and needles.

LJ agar showed yellowish white, slightly elevated colonies after 5 days of incubation at $37^{\circ} \mathrm{C}$. ZN staining showed presence of long, filamentous acid fast bacilli in clumps.

Growth on Blood agar plate was sent to reference laboratory where it was identified as Mycobacterium abscessus. The identification was carried out by MALDITOF-MS (Matrix Assisted Laser Desorption Ionisation - Mass Spectrometry).

The patient had no history of any immunocompromised condition and he was not on any immunosuppressive medications. The patient was a known diabetic, with HbAlc level of 9, was on treatment for the same. His Lipid profile was unremarkable. His hematologic work up was normal except for mildly elevated Erythrocyte Sedimentation Rate i.e. 12. Liver Function Tests and Renal Function Tests were within normal limits. Serum electrolytes were in normal range. Thyroid Function Tests were normal. Prostate Specific Antigen (PSA) and Carcinoembryonic Antigen (CEA) were not elevated. 
The isolate was reported as Non Tuberculous Mycobacterium and results were informed to orthopedician. After drainage of fluid, patient had recovered and did not show any recurrent symptoms. The patient was asked to follow up if any clinical symptoms develop (Fig. 17).

Fig.1 Turbidity in MGIT broth

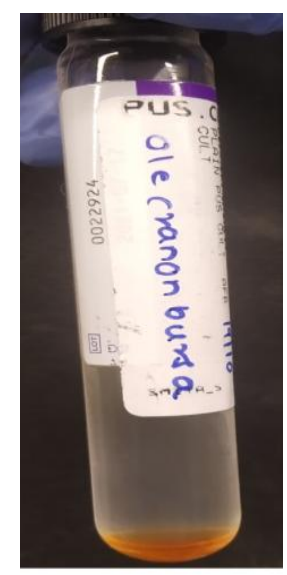

Fig.2 ZN stained smear from MGIT broth

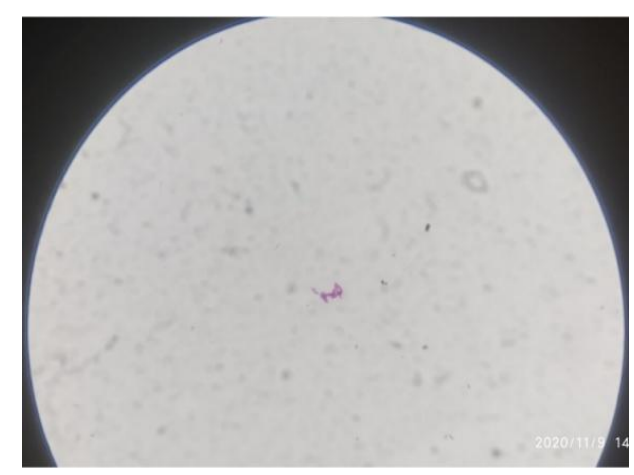

Fig.3 Growth of NTM (M. abscessus) on Blood Agar

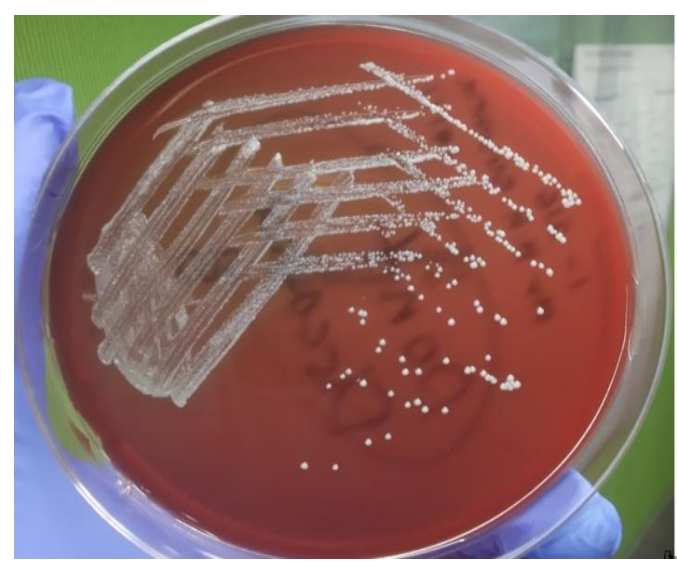


Fig.4 Gram stain of growth from Blood agar plate

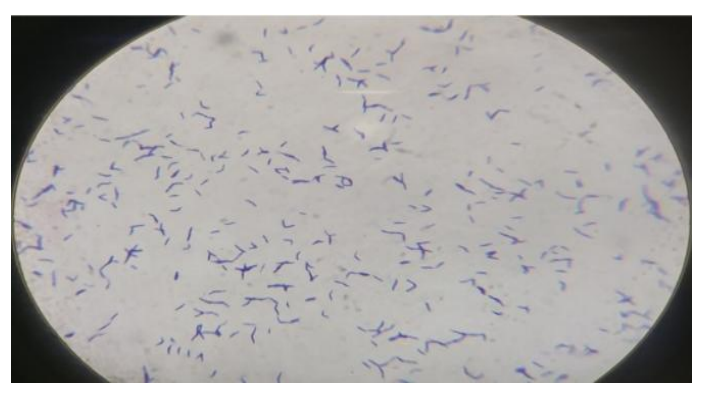

Fig.5 ZN stain of growth from Blood agar plate

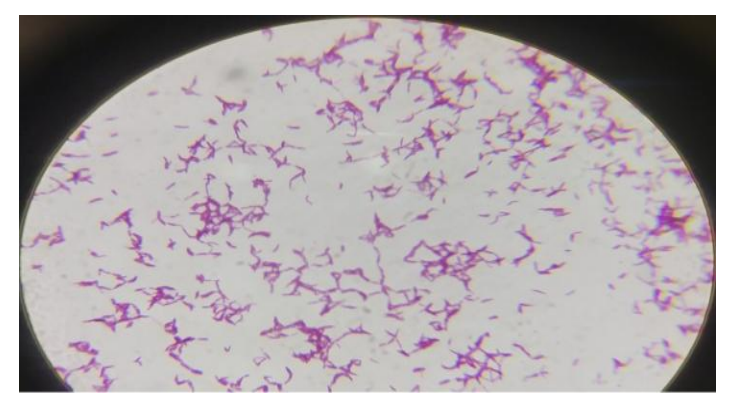

Fig.6 Growth of NTM (M. abscessus) on LJ medium

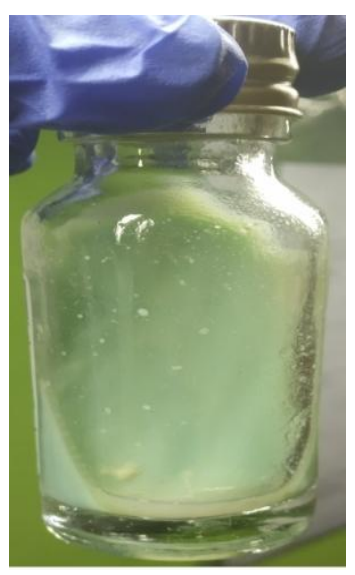

Fig.7 ZN staining of smear from LJ agar growth

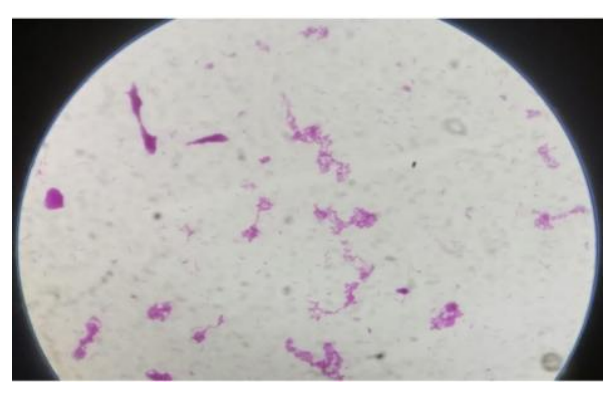


Nontuberculous mycobacteria are isolated from a wide variety of environmental sources including soil, water, dust, Sphagnum vegetation which might colonize and cause infection in humans and animals. These infections are increasingly becoming prevalent in immunocompromised hosts, e.g. HIV, lymphoproliferative disorders or transplants, and those on immunosuppressive therapy (Brown-Elliott and Wallace. 2002).

In transplant patients, most NTM infections are chronic infections of soft tissues and joints (cutaneous lesions on the extremities, tenosynovitis, arthritis) and osteomyelitis, often with multifocal involvement. Constitutional symptoms are absent. Most commonly involved joints are carpometacarpal, wrist, elbow, ankle and knee. In the immunocompetent host, NTM can cause infections in cutaneous, deep soft tissues, lymphatics, and other sites (e.g. skeletal, peritoneal catheter-related, ocular).

Mycobacterium abscessus is a rapidly growing non-tuberculous mycobacterium which is an emerging pathogen worldwide. The earliest case of $M$. abscessus was reported in 1951 and described infection associated with traumatic knee injury. Until recently, $M$. abscessus was considered a subspecies of Mycobacterium chelonae. In 1992 genetic analysis demonstrated that it was a distinct species and it was elevated to species status. (Ryan and Byrd, 2018)

Mycobacterium abscessus is implicated in both pulmonary and extra-pulmonary infections, underlying risk factors for infections include organ transplantation, rheumatoid arthritis and other autoimmune disorders, trauma, including invasive medical procedures. Patients with underlying structural lung disease (prior tuberculosis, bronchiectasis, cystic fibrosis) are prone to pulmonary infection with $M$. abscessus, which is difficult to treat (Griffith, et al., 2007). M. abscessus has also increasingly been reported to cause CNS infections in HIV-negative patients, especially in those who had undergone neurosurgical procedures, indwelling intracranial catheters and otologic diseases. (Lee, et al., 2012) Conjunctivitis, scleritis, keratitis (in hard and soft contact lens wearers) and endophthalmitis have also been reported (Girgis et al., 2012).

The most common site of extra-pulmonary infections being skin and subcutaneous tissues. (Brown-Elliott BA and Wallace Jr RJ, 2002). Infections are reported following direct inoculation, through contaminated acupuncture needles, injection solutions, and surgical procedures or as a result contamination of traumatic wound (Scholze et al., 2005; Ryu et al., 2005; Yuan et al., 2009; Schnabel et al., 2016).

Disseminated $M$. abscessus infections have been seen in both immunocompromised as well as immunocompetant patients (MengRui Lee et al., 2015)

Our patient reported swelling, erythema and tenderness over left elbow one and half month following injury to the elbow. Though our patient had no history of any immunosuppressive condition, he was diabetic which could have contributed as a risk factor for developing an infection. Nontuberculous mycobacteria infections are rare, indolent, and frequently misidentified. Discrimination between colonization and infection by NTM can be difficult.

Many cases of olecranon bursitis caused by nontuberculous mycobacteria (M. avium complex, M. marinum, $M$. asciaticum, $M$. chelonae, $M$. fortuitum, $M$. gordonae, $M$. Kansasii, M. szulgai, M. terrae, M. goodie and $M$. longobardum) have been reported in literature which were treated with 
antimycobacterial therapy alone or with surgical intervention (Working et al., 2015).

Two cases of olecranon bursitis caused by $M$. lucas and M. szulgai are also reported to be treated solely by surgical intervention (Turenne et al., 2002; Marks et al., 1972). Some minor infections caused by $M$. abscessus species can resolve spontaneously or with surgical debridement.

For serious skin, soft tissue and bone infections caused by $M$. abscessus, clarithromycin or azithromycin should be combined with parenteral medications (amikacin, cefoxitin, or imipenem). For bone infections, 6 months of therapy is recommended whereas for serious infections, minimum 4 months of therapy is advised (Griffith et al., 2007).

Given our patient's immunocompetant status and early surgical drainage of olecranon bursa fluid, he did not have any recurrence of symptoms. He was not advised antimycobacterial therapy by treating clinician.

In conclusion the nontuberculous mycobacteria infections could be challenging to diagnose, treat and to follow up with. Occurrence of NTM in a clinical specimen should not be ignored as contamination and should always be clinically correlated. In the present study case shows that NTM infections can take place in immunocompetant hosts also, given the ubiquitous presence of mycobacteria in surrounding environment. In case of recurrence of symptoms or lymphatic spread of mycobacteria if any, happened before surgical intervention, which could give rise to disseminated disease, diagnosis and treatment will be rapid due to identification of primary isolate in our patient.

\section{References}

Brown-Elliott BA, Wallace Jr RJ. Clinical and taxonomic status of pathogenic nonpigmented or late-pigmenting rapidly growing mycobacteria. Clinical Microbiol Rev. 2002; 15(4):716-46.

Girgis DO, Karp CL, Miller D. Ocular infections caused by non-tuberculous mycobacteria: update on epidemiology and management. Clin Experiment Ophthalmol. 2012;40:467-75.

Griffith DE, Aksamit T, Brown-Elliott BA, Catanzaro A, Daley C, Gordin F, et al., An official ATS/IDSA statement: diagnosis, treatment, and prevention of nontuberculous mycobacterial diseases. Am J Respir Crit Care Med. 2007; 175: 396-7.

Lee MR, Cheng A, Lee YC, Yang CY, Lai CC, Huang YT, et al., CNS infections caused by Mycobacterium abscessus complex: clinical features and antimicrobial susceptibilities of isolates. J Antimicrob Chemother. 2012; 67: 222-5.

Marks J, Jenkins PA, Tsukamura M. Mycobacterium szulgai - a new pathogen. Tubercle 1972; 53(3): 210-4.

Meng-Rui Lee, Wang-Huei Sheng, ChienChing Hung, Chong-Jen Yu, Li-Na Lee, Po-Ren Hsueh. Mycobacterium abscessus Complex Infections in Humans. Emerging Infectious Diseases. 2015; 21(9):1638-46.

Ryan K and Byrd TF. Mycobacterium abscessus: Shapeshifter of the Mycobacterial World. Frontiers in Microbiology. 2018;9:2642.

Ryu H. J., Kim W. J., Oh C. H., and Song H. J. Iatrogenic Mycobacterium abscessus infection associated with acupuncture: clinical manifestations and its treatment. Int. J. Dermatol. 2005; 44: 846-850.

Schnabel D., Esposito D. H., Gaines J., Ridpath A., Barry M. A., Feldman K. 
A., et al., Multistate US outbreak of rapidly growing mycobacterial infections associated with medical tourism to the Dominican republic, 2013- 2014(1). Emerg. Infect. Dis. 2016;22: 1340-47.

Scholze A., Loddenkemper C., Grunbaum M., Moosmayer I., Offermann G., and Tepel M. Cutaneous Mycobacterium abscessus infection after kidney transplantation. Nephrol. Dial. Transplant. 2005; 20: 1764-65.

Turenne C, Chedore P, Wolfe J, Jamieson F, Broukhanski G, May $\mathrm{K}$, et al., Mycobacterium lacus sp. nov., a novel slowly growing, non-chromogenic clinical isolate. Int $\mathrm{J}$ Syst Evol Microbiol 2002; 52(Pt 6): 2135-40.

Working S, Tyser A, Levy D. Mycobacterium avium complex olecranon bursitis resolves without antimicrobials or surgical intervention: A case report and review of the literature. IDCases. 2015; 2: 59-62.

Yuan J., Liu, Y., Yang, Z., Cai, Y., Deng, Z., Qin, P., et al., Mycobacterium abscessus post-injection abscesses from extrinsic contamination of multiple dose bottles of normal saline in a rural clinic. Int. J. Infect. Dis. 2009; 13: 537-542.

\section{How to cite this article:}

Sneha Bowalekar and Rahul Gadkari. 2021. A Rare Case of Olecranon Bursitis caused by Mycobacterium abscessus Treated with Surgical Intervention. Int.J.Curr.Microbiol.App.Sci. 10(02): 476-482. doi: https://doi.org/10.20546/ijcmas.2021.1002.056 